\title{
Méthode d'analyse des pluies pour l'évaluation de l'impact de la pollution générée par le ruissellement urbain
}

\author{
Rainfall analysis method for the evaluation of the pollution \\ impact generated by urban runoff
}

\author{
par \\ Catherine Savart, Louis Herremans, Christophe Zobrist \\ Anjou Recherche, Branche "Assainissement" \\ et \\ Christophe Merlier \\ Météo-France, Service Central d'Exploitation de la Météorologie,
}

A method of rainfall data analysis was defined to study the impact of the pollution due to urban stormwater nunoff on receiving waters. The available pluviographic records were analysed to draw up an inventory of the rainy events over the observation period. An automatic classification was then used to obtain a typology of the events. At last a synthetic year made of rainfalls generating runoff was produced on the basis of the different classes previously defined.

\section{INTRODUCTION}

Une étude initiée par l'Agence de l'Eau Rhin-Meuse est actuellement menée dans la vallée de l'Orne. Celle-ci se situe en région Lorraine, c'est-à-dire dans le nord-est de la France, et est caractérisée par un climat à tendance continentale. Il s'agit d'évaluer l'impact de la pollution générée par le ruissellement urbain sur le milieu récepteur, en termes d'effets aigus, chroniques et cumulatifs.

Dans le cadre de cette réflexion, les données pluviométriques doivent permettre de dégager les événements pluvieux à mettre en relation avec ces différents niveaux de pollution. Or, l'approche française classique du traitement des pluies en termes d'occurrence ou de période de retour était à écarter, en raison de son inadéquation avec les objectifs de l'étude.

Dans ce cadre d'hydrologie urbaine et en l'absence d'approche similaire connue pour traiter l'évaluation de l'impact de la pollution générée par le ruissellement urbain, une méthode d'analyse originale a été mise au point afin, d'une part, de sélectionner des événements types à caractère exceptionnel, susceptibles d'entraîner des effets aigus dans la rivière et, d'autre part, de définir une année pluviographique synthétique, caractérisée par la succession d'événe- ments pluvieux et de périodes de temps sec, permettant de mettre en évidence les effets chroniques et cumulatifs.

La méthodologie et les résultats des traitements statistiques sont présentés dans cet article.

\section{II — CADRE GÉNÉRAL}

\subsection{Approche retenue}

Les traitements statistiques se sont déroulés de la façon suivante :

- première étape: définition précise des éléments de l'étude (terminologie, période d'observation, individus statistiques...) ;

- deuxième étape: analyse du temps de pluie, qui consiste à inventorier les événements enregistrés au cours de la période d'observation, puis à les classifier ;

- troisième étape: traitement des durées de temps sec précédant un événement pluvieux, afin d'observer un lien éventuel entre leur répartition et la classe à laquelle appartient l'événement ; 
- quatrième étape: détermination d'une année synthétique à partir des résultats obtenus lors des phases précédentes.

\subsection{Terminologie adoptée}

Chacun des termes a été défini au vu des objectifs généraux de l'étude et des caractéristiques de la zone concernée.

- "Épisode pluvieux" : intervalle de temps d'intensité pluvieuse constante, qui constitue la donnée de base des enregistrements pluviographiques de Météo-France.

— "Événement pluvieux" : somme d'épisodes pluvieux consécutifs dont le cumul est supérieur à $2 \mathrm{~mm}$ et dont la fin est fixée par une interruption des précipitations d'au moins 30 minutes (épisode sec durant au moins $30 \mathrm{mn}$ ).

— "Temps sec au sens strict" : noté TSS, correspond à la durée de temps sec précédant immédiatement l'événement pluvieux.

— "Temps sec au sens large" : noté TSL, correspond à la durée de temps sec précédant immédiatement l'événement pluvieux, augmentée de la durée des épisodes n'entrant pas dans la définition d'un événement pluvieux car ne répondant pas aux critères fixés plus haut.

\section{- 2.3. Données disponibles}

\section{- Poste d'observation}

Il s'agit du pluviographe situé sur l'aéroport de Metz-Frescaty. Ce poste a été choisi en raison de sa proximité par rapport à la zone d'étude (vallée de l'Orne).

\section{- Période d'observation}

La période d'observation a été déterminée par la qualité des enregistrements pluviographiques. Les traitements statistiques envisagés imposent en effet de travailler à partir d'enregistrements chronologiques continus, c'est-à-dire sur une période ne présentant pas ou peu d'épisodes manquants. La période retenue sur la base de ce critère est relativement courte, puisqu'elle ne réunit que 6 années (de janvier 1982 à décembre 1987). Les années postérieures n'ont pu être traitées du fait de leur qualité (discontinuité de la série). Cependant, cela n'est pas très grave puisque le but poursuivi est ici surtout de mettre au point une méthodologie adaptée au problème envisagé et transposable à d'autres sites.

\section{- Période de traitement}

Le traitement a été effectué sur trois périodes distinctes : la période totale (l'année entière), la période dite "normale" (d'octobre à avril inclus) et la période dite "critique" (de mai à septembre inclus). Cette distinction est basée sur le régime hydrologique du cours d'eau étudié, dont la période d'étiage a lieu de mai à septembre. C'est en effet à cette époque de l'année que le cours d'eau est le plus vulnérable face à une éventuelle pollution.

\section{III — TRAITEMENT DU TEMPS DE PLUIE}

\subsection{Inventaire des événements pluvieux}

Chaque événement inventorié est décrit par :

- sa date de début, repérée par l'année, le mois, le jour,

l'heure et les minutes ;

- sa durée $(d)$, en minutes ;

- sa hauteur totale $(h)$, en millimètres ;

- sa durée de temps sec au sens strict (TSS), en minutes ;

- sa durée de temps sec au sens large (TSL), en minutes.

L'inventaire a permis de mettre en évidence 428 événements sur l'ensemble de la période d'observation, 218 se produi- sant entre octobre et avril inclus (période normale) et 210 ayant lieu au cours de la période critique (mai à septembre inclus).

\subsection{Classification des événements pluvieux}

Le principe consiste à regrouper les événements pluvieux recensés précédemment, sur la valeur du couple $(h, d)$, de manière à obtenir des classes les plus compactes et les plus distantes possible.

\section{- Analyse graphique}

L'analyse graphique du nuage de points d'abscisse $d$ et d'ordonnée $h$, tracé pour les trois périodes (totale, normale et critique) a été effectuée dans un premier temps, afin de dégager les tendances générales.

A l'observation, il s'est avéré que la quasi totalité des valeurs était simultanément faible pour $h$ comme pour $d$, rendant tout regroupement graphique impossible.

Le recours à un outil statistique plus complexe était donc indispensable pour effectuer la classification des événements pluvieux.

\section{- Classification automatique}

Les variables $h$ et $d$ n'étant pas homogènes du point de vue dimensionnel ( $d$ est exprimée en minutes et $h$ en millimètres), leurs plages de variations sont très différentes. Il n'est donc pas possible de définir la distance entre deux événements par la distance euclidienne comme il est classique de le faire d'une manière générale entre deux éléments $(i, j)$. Cette distance se note $\delta(i, j)$ et s'exprime par :

$\delta^{2}(i, j)=\left(d_{i}-d_{\mathrm{j}}\right)^{2}+\left(h_{i}-h_{\mathrm{j}}\right)^{2}$

Afin de s'affranchir de ces problèmes de dimension, les variables ont été réduites par la valeur maximale observée de l'échantillon. Cette méthode permet d'obtenir la formulation suivante pour la définition de la distance réduite entre les deux variables :

$$
\delta^{2}(i, j)=\left(\frac{d_{i}-d_{j}}{d_{\max }}\right)^{2}+\left(\frac{h_{i}-h_{j}}{h_{\max }}\right)^{2}
$$

Cette normalisation par division par la valeur maximale permet de ramener les deux variables dans le même intervalle $[0,1]$ (ceci n'est pas du tout le cas si l'on norme par la valeur moyenne - voir tableau 1 pour les ordres de grandeur).

Le type de classification adopté est une classification hiérarchique ascendante. Le critère d'agglomération de Ward a été utilisé afin de minimiser la variance intraclasse et maximiser la variance interclasse. Ce critère est l'un des plus répandus en matière de classification, du moins en météorologie, car il a l'avantage de produire des classes à la fois très compactes (ramassées sur elles-mêmes : variance intraclasse minimale) et très bien différenciées les unes des autres (variance interclasse maximale).

Le traitement a été effectué sur les trois périodes (totale, normale et critique), ce qui a consisté à classer successivement 428,218 puis 210 événements.

On obtient grâce à cette procédure 9 classes pour la période totale et 7 classes pour chacune des deux autres périodes.

Cette classification a permis de mettre en évidence plusieurs types d'événements pluvieux. Ceux-ci sont décrits par différents paramètres descriptifs des variables utilisées : minimum, maximum, moyenne, écart-type et fréquences d'occurrence (séparées en quintiles). Le tableau 1 fait la synthèse de ces éléments descriptifs pour la période totale. La figure 1 représente les éléments moyens pour les 9 classes déterminées pour la période totale. 


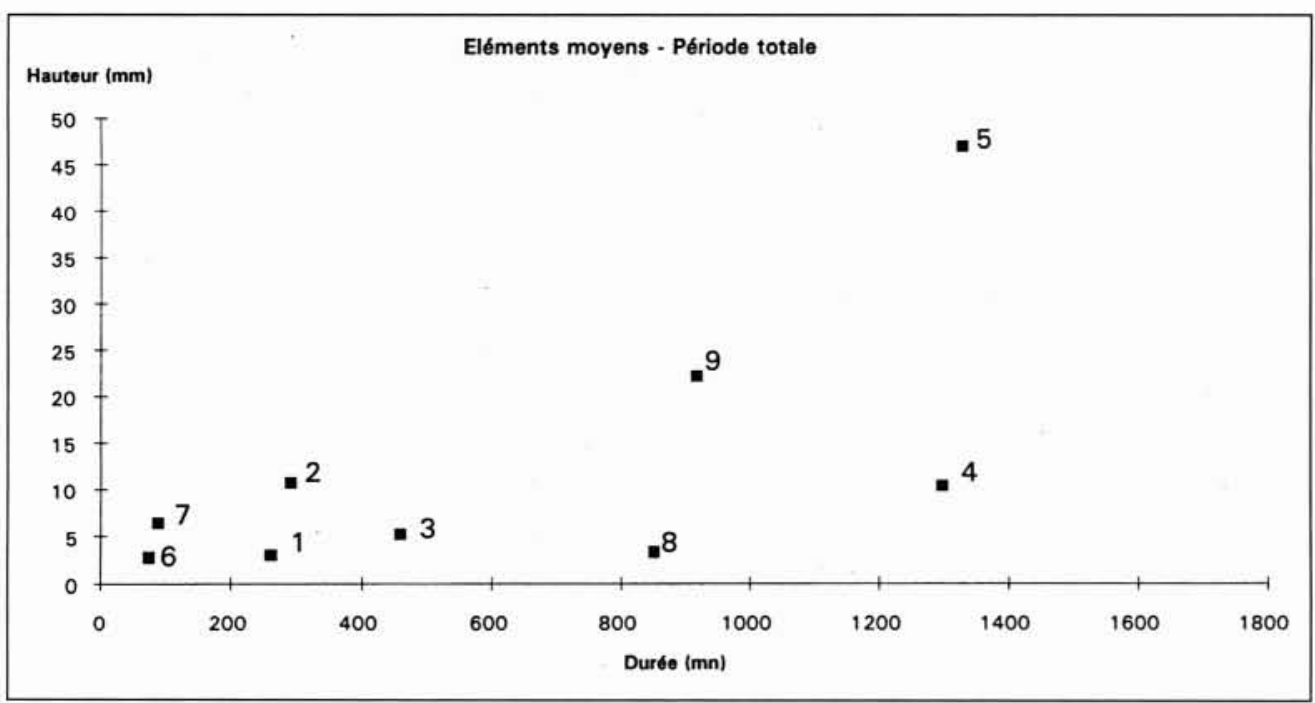

1. Pointage des événements moyens des classes pour la période totale

On distingue ainsi pour les trois périodes analysées :

- Type 1: Courtes durées $(\approx 100 \mathrm{mn})$, hauteurs faibles $(\approx 5 \mathrm{~mm})$

- classes 6 et 7 de la période totale, qui regroupent au total $45 \%$ des événements ;

- classe 4 de la période normale $(51 \%$ des événements) ;

- classes 3 et 7 de la période critique.

- Type 2: Courtes durées (200 mn), hauteurs plus élevées à fortes $(<10 \mathrm{~mm})$;

- classes 1 et 2 de la période totale (1/3 des événements) ;

- classe 2 de la période normale $(12 \%$ des événements) ;

- classe 2 et 7 de la période critique $(30 \%$ des événements).
- Type 3 : Longues durées ( $>600 \mathrm{mn}$ ), hauteurs faibles (4 mm) ;

- classe 8 de la période totale, $65 \%$ des cas ;

- classe 1 de la période normale, $6 \%$ des cas ;

- classes 1 et 4 de la période critique, qui regroupent respectivement 1,5 et $10,5 \%$ des cas.

- Type 4 : Longues durées (>1000 mn), hauteurs fortes (> $10 \mathrm{~mm})$.

- classes 4,5 et 9 de la période totale ( $5 \%$ des événements) ;

- classes 3 et 7 de la période normale $(<5 \%$ des événements) ;

- classes 5 et 6 de la période critique $(<5 \%$ des événements).

\begin{tabular}{|c|c|c|c|c|c|c|c|c|c|c|c|}
\hline Classe & nb & var. & min. & max. & m & $\sigma$ & $\mathbf{q 5 0}$ & $\mathbf{q 2 0}$ & $\mathbf{q 4 0}$ & $\mathbf{q 6 0}$ & $\mathbf{q 8 0}$ \\
\hline $\mathbf{1}$ & 109 & d & 134,0 & 541,0 & 261,4 & 88,1 & 240,0 & 183,0 & 213,0 & 265,0 & 334,0 \\
\hline & & h & 2,0 & 5,1 & 3,0 & 0,8 & 2,7 & 2,2 & 2,5 & 3,0 & 3,8 \\
\hline $\mathbf{2}$ & 32 & d & 8,0 & 576,0 & 291,4 & 145,6 & 294,0 & 199,6 & 248,2 & 307,6 & 446,0 \\
\hline & & h & 7,2 & 16,8 & 10,7 & 2,7 & 10,4 & 8,1 & 9,1 & 10,6 & 13,2 \\
\hline $\mathbf{3}$ & 48 & d & 249,0 & 733,0 & 458,7 & 126,0 & 450,5 & 342,4 & 416,2 & 500,4 & 561,0 \\
\hline & & h & 3,2 & 8,5 & 5,2 & 1,2 & 5,0 & 4,2 & 4,8 & 5,4 & 6,2 \\
\hline $\mathbf{4}$ & 15 & d & 745,0 & 1741,0 & 1298,3 & 333,3 & 1346,0 & 926,4 & 1165,8 & 1562,8 & 1610,4 \\
\hline & & h & 5,0 & 14,2 & 10,4 & 2,4 & 9,9 & 8,5 & 9,3 & 11,3 & 12,8 \\
\hline $\mathbf{5}$ & $\mathbf{1}$ & d & 1329,0 & 1329,0 & 1329,0 & - & - & - & - & - & \\
\hline & & h & 46,9 & 46,9 & 46,9 & - & - & - & - & - & - \\
\hline $\mathbf{6}$ & 146 & d & 7,0 & 170,0 & 74,8 & 41,7 & 70,0 & 31,4 & 53,0 & 87,2 & 119,0 \\
\hline & & h & 2,0 & 4,3 & 2,7 & 0,6 & 2,5 & 2,1 & 2,3 & 2,7 & 3,4 \\
\hline $\mathbf{7}$ & 45 & d & 10,0 & 203,0 & 89,0 & 50,9 & 90,0 & 39,2 & 64,4 & 103,0 & 141,4 \\
\hline & & h & 4,7 & 8,8 & 6,4 & 1,2 & 6,2 & 5,2 & 5,8 & 6,4 & 7,5 \\
\hline $\mathbf{8}$ & 28 & d & 578,0 & 1331,0 & 851,6 & 228,8 & 789,0 & 659,6 & 708,6 & 837,8 & 1130,6 \\
\hline & & h & 2,0 & 6,2 & 3,3 & 1,1 & 2,8 & 2,4 & 2,7 & 3,2 & 4,3 \\
\hline $\mathbf{9}$ & $\mathbf{4}$ & d & 587,0 & 1150,0 & 917,5 & 207,0 & - & - & - & & - \\
\hline & & h & 19,4 & 23,4 & 22,1 & 1,6 & - & - & - & - & - \\
\hline
\end{tabular}

Tableau 1: Paramètres descriptifs pour les différentes classes des variables $h$ et $d$ pour la période totale. 


\section{IV — TRAITEMENT DU TEMPS SEC}

\subsection{Objectif}

L'objectif est d'étudier la répartition des durées de temps sec précédant un événement pluvieux, pour les différentes classes mises en évidence précédemment, afin de voir s'il existe un lien entre cette distribution et la classe à laquelle appartient l'événement.

\section{- 4.2. Méthodologie}

Les variations de TSS et de TSL ont été réparties en 5 intervalles de fréquence équivalente (20\%) selon des quintiles de distribution, au sein des différentes périodes.

Pour chaque classe, on dispose du nombre de cas correspondant aux 5 intervalles définis précédemment, soit 5 valeurs $\left(n_{i}\right)_{i=1,5}$ décrivant la répartition des valeurs de TSS et de TSL.

La variable $Y^{2}=\sum_{i=1}^{5} \frac{\left(n_{i}-N / 5\right)^{2}}{N / 5} \quad$ (où $N=\sum_{i=1}^{5} n_{i}$ ) suit une loi du $\chi^{2}$ à $v=5-1=4$ degrés de liberté.

La valeur $N / 5$ correspond au nombre théorique d'observations que l'on aurait si la distribution se faisait au hasard, c'est-à-dire si la distribution au sein d'une classe était la même que celle observée pour la totalité de l'échantillon.

Pour un couple de valeurs $\left(Y^{2}, v\right)$, on peut calculer la probabilité, notée $p$, correspondant à la valeur observée de $Y^{2}$, d'après la loi théorique de distribution de $Y^{2}$. En fonction de la valeur de cette probabilité, on peut donc avoir une idée de la façon dont sont distribuées les occurrences de TSS et de TSL et conclure si elles le sont ou non au hasard.

\section{- 4.3. Résultats}

On constate à plusieurs reprises (voir résultats plus loin), que la répartition des TSS et/ou des TSL est biaisée (valeur de $p>90 \%$ ), c'est-à-dire que les TSS et les TSL sont plus longues ou plus courtes que celles déterminées sur la totalité de l'échantillon.

Il apparaît donc que la distribution des périodes de temps sec n'est pas aléatoire pour toutes les classes.

A titre d'illustration, on peut considérer l'exemple suivant. Pour les classes 6 et 7 de la période totale, on obtient des TSS particulièrement faibles (les valeurs des TSS sont plus faibles que celles de la totalité de l'échantillon). Cela pourrait s'expliquer par le fait que ce type d'événements correspond en fait plutôt à des averses se produisant dans des situations de traîne (temps instable à l'arrière d'une perturbation). La durée de temps sec entre deux événements consécutifs est alors particulièrement courte. Cette assertion semble par ailleurs corroborée par l'observation des TSL, pour lesquels aucune structure particulière n'est détectée $(p$ de l'ordre de 55\%).

Ces informations viennent compléter les données déjà collectées pour chaque classe concernant la pluviographie (tableau 2).

\section{V — DÉTERMINATION D'UNE "ANNÉE SYNTHÉTIQUE"}

\subsection{Objectif}

Afin de pouvoir utiliser les résultats précédents pour simuler l'impact des précipitations sur la pollution générée par le ruissellement, on a cherché à reproduire une année "synthétique " en ce sens qu'elle représente correctement les précipitations au sens de divers critères. L'objectif est donc d'obtenir une série chronologique correspondant à une année, constituée de valeurs pour les variables $d$ (durée de l'événement), $h$ (hauteur précipitée pendant l'événement) et TSL (durée de temps sec le précédant), représentatives de la pluviométrie annuelle de la station considérée.

\begin{tabular}{|c|c|c|c|c|c|c|c|c|c|c|c|c|}
\hline Classe & $\mathbf{n}_{\mathbf{1}}$ & & $\mathbf{C}_{\mathbf{1}}$ & & $\mathbf{n}_{\mathbf{2}}$ & & $\mathbf{C}_{\mathbf{2}}$ & & $\mathbf{n}_{\mathbf{3}}$ & & $\mathbf{C}_{\mathbf{3}}$ & \\
\hline & TSS & TSL & TSS & TSL & TSS & TSL & TSS & TSL & TSS & TSL & TSS & TSL \\
\hline $\mathbf{1}$ & 14 & 16 & 2,8 & 1,5 & 20 & 24 & 0,1 & 0,2 & 19 & 28 & 0,4 & 1,8 \\
\hline $\mathbf{2}$ & 4 & 2 & 0,9 & 3,0 & 6 & 10 & 0,0 & 2,0 & 9 & 8 & 1,1 & 0,4 \\
\hline $\mathbf{3}$ & 6 & 9 & 1,3 & 0,0 & 8 & 14 & 0,3 & 2,0 & 11 & 8 & 0,2 & 0,3 \\
\hline $\mathbf{4}$ & 3 & 3 & 0,0 & 0,0 & 3 & 4 & 0,0 & 0,3 & 3 & 3 & 0,0 & 0,0 \\
\hline $\mathbf{5}$ & 0 & 0 & 0,2 & 0,2 & 0 & 1 & 0,2 & 3,2 & 0 & 0 & 0,2 & 0,2 \\
\hline $\mathbf{6}$ & 38 & 38 & 2,7 & 2,7 & 37 & 26 & 2,1 & 0,4 & 27 & 24 & 0,2 & 0,9 \\
\hline $\mathbf{7}$ & 14 & 12 & 2,8 & 1,0 & 5 & 6 & 1,8 & 1,0 & 13 & 6 & 1,8 & 1,0 \\
\hline $\mathbf{8}$ & 6 & 5 & 0,0 & 0,1 & 6 & 1 & 0,0 & 3,8 & 2 & 7 & 2,3 & 0,4 \\
\hline $\mathbf{9}$ & 0 & 0 & 0,8 & 0,8 & 1 & 0 & 0,1 & 0,8 & 2 & 2 & 1,8 & 1,8 \\
\hline
\end{tabular}

\begin{tabular}{|c|c|c|c|c|c|c|c|c|c|c|c|c|c|}
\hline Classe & $\mathbf{n}_{\mathbf{4}}$ & & $\mathbf{C}_{\mathbf{4}}$ & & $\mathbf{n}_{\mathbf{5}}$ & & $\mathbf{C}_{\mathbf{5}}$ & & $\mathbf{Y}^{\mathbf{2}}$ & & $\mathbf{p}$ & & $\mathbf{N}$ \\
\hline & TSS & TSL & TSS & TSL & TSS & TSL & TSS & TSL & TSS & TSL & TSS & TSL & \\
\hline $\mathbf{1}$ & 34 & 20 & 6,8 & 0,1 & 22 & 21 & 0,0 & 0,0 & 10,1 & 3,7 & 0,96 & 0,55 & 109 \\
\hline $\mathbf{2}$ & 8 & 7 & 0,4 & 0,1 & 5 & 5 & 0,3 & 0,3 & 2,7 & 5,8 & 0,39 & 0,79 & 32 \\
\hline $\mathbf{3}$ & 12 & 8 & 0,6 & 0,3 & 11 & 9 & 0,2 & 0,0 & 2,6 & 2,6 & 0,38 & 0,38 & 48 \\
\hline $\mathbf{4}$ & 3 & 2 & 0,0 & 0,3 & 3 & 3 & 0,0 & 0,0 & 0,0 & 0,7 & 0,00 & 04 & 15 \\
\hline $\mathbf{5}$ & 1 & 0 & 3,2 & 0,2 & 0 & 0 & 0,2 & 0,2 & 4,0 & 4,0 & 0,59 & 59 & 1 \\
\hline $\mathbf{6}$ & 18 & 29 & 4,3 & 0,0 & 26 & 29 & 0,4 & 0,0 & 9,5 & 3,9 & 0,95 & 58 & 146 \\
\hline $\mathbf{7}$ & 4 & 10 & 2,8 & 0,1 & 9 & 11 & 0,0 & 0,4 & 9,1 & 3,6 & 0,94 & 53 & 45 \\
\hline $\mathbf{8}$ & 6 & 9 & 0,0 & 2,1 & $\mathbf{8}$ & 6 & 1,0 & 0,0 & 3,4 & 6,3 & 0,51 & 82 & $\mathbf{2 8}$ \\
\hline $\mathbf{9}$ & 0 & 1 & 0,8 & 0,1 & 1 & 1 & 0,1 & 0,1 & 3,5 & 3,5 & 0,52 & 52 & 4 \\
\hline
\end{tabular}

Tableau 2: Analyse des durées de temps sec pour la période totale (TS et TSL). 


\subsection{Méthodologie}

La chronologie des événements est créée à partir d'un processus itératif (de type "générateur de séries climatiques "). Le traitement débute le ler janvier à 00 h00.

\section{- Détermination de la classe d'appartenance de l'événe- ment à générer}

L'affectation de l'événement à une classe se déroule en deux étapes :

- tirage au sort d'une valeur dans l'intervalle $[0,1]$,

- détermination de la classe correspondant à cette valeur (avec prise en compte de la date courante pour détermination de la période) en fonction des probabilités d'occurrence des différentes classes.

\section{- Détermination des valeurs des variables $d, h$ et $T S L$ pour une classe donnée}

\section{Principe général}

On dispose par classe, des distributions de ces variables, c'est-à-dire de toutes les valeurs prises par ces variables au sein de la classe. Un tirage au sort est effectué de la même manière que précédemment. Une valeur est ainsi prise au hasard dans l'intervalle $[0,1]$, puis on détermine la valeur de la variable dont la fréquence cumulée d'apparition correspond à cette valeur tirée au sort.

Une telle démarche suppose que les variables sont indépendantes au sein de chaque classe.

Une étude des liens entre les variables d'une même classe a donc été effectuée.

Une première analyse graphique du nuage de points de coordonnées $(d, h),(d, T S L)$ et $(h, T S L)$ a permis de représenter visuellement les éventuelles liaisons. Le calcul de corrélations entre ces couples de variables a confirmé les tendances observées par une analyse graphique. Dans le tableau 3, sont indiquées les valeurs des corrélations entre les couples de variables mises en jeu.

Dans le cas où un lien a été établi entre deux variables, les deux valeurs ne sont pas tirées au sort : la deuxième variable est estimée une fois que la première a été déterminée.

\section{Évaluation de la valeur de d}

- Tirage au sort d'une valeur dans l'intervalle $[0,1]$.

- Détermination de la valeur de $d$ calculée, en tenant compte de la répartition de cette variable au sein de la classe.

Évaluation de la valeur de $h$

On distingue deux cas :

- Une liaison a été mise en évidence entre $d$ et $h$ pour la classe considérée, la valeur de $h$ est alors déterminée en prenant en compte cette liaison.
- Aucune liaison ne semble exister : on détermine alors $h$ de la même façon que $d$, c'est-à-dire de manière purement aléatoire.

Évaluation de la valeur de TSL

Le traitement est identique à celui retenu pour l'évaluation de $h$, à la différence que les éventuelles liaisons constatées entre TSL et $d$ ou TSL et $h$ sont prises en compte.

Ces différents paramètres étant déterminés, il reste à définir la date (mois, jour, heure) de la fin de chaque événement. On retourne ensuite à la première étape, qui correspond à la détermination de la classe de l'événement suivant, tant que l'on n'a pas atteint le $31 / 12$ à $23 \mathrm{~h} 59$.

\subsection{Critères de validation}

La méthodologie présentée permet de produire un certain nombre d'années "synthétiques". Il est alors judicieux de ne retenir que celle qui semble la plus représentative de la pluviométrie de la station considérée. Un critère de qualité a donc été appliqué sur l'ensemble des années produites. $\mathrm{Ce}$ critère s'appuie sur les paramètres suivants :

- le nombre total d'événements ;

- la durée totale de précipitations ;

- la hauteur totale de précipitations ;

- la durée totale de temps sec au sens large (TSL).

Les quintiles correspondant aux fréquences de non-dépassement de $20,40,45,50,55,60$ et $80 \%$ ont été calculés pour ces quatre paramètres, sur les 6 années d'observation.

L'année-type devant être proche d'une année "médiane", il fallait alors rechercher l'année qui présentait des valeurs des quatre paramètres considérées comme peu éloignées des valeurs médianes $(q 50)$. Deux années ont été comparées, la première étant caractérisée par des valeurs comprises, pour les quatre paramètres, entre les quintiles $q 40$ et $q 60$ et la seconde par des valeurs comprises entre les quintiles $q 45$ et $q 55$.

La seconde année a finalement été sélectionnée. Elle présentait, d'une part, une meilleure répartition du nombre d'événements à l'intérieur des deux périodes (39 en période normale et 37 en période critique) et, en ce sens, se rapproche plus d'une année standard, qui voit ses événements équirépartis entre les deux périodes. Elle offrait, d'autre part, une meilleure adéquation à la répartition fréquentielle des événements au sein des différentes classes pour une période donnée.

\subsection{Résultats et commentaires}

L'année synthétique ainsi constituée se compose de 76 événements (voir figure 2).

La hauteur cumulée des précipitations est de $333,6 \mathrm{~mm}$, ce qui représente une valeur beaucoup plus faible que le cumul annuel médian de précipitations réelles (de l'ordre de $730 \mathrm{~mm}$ ). Cela s'explique par le fait que tous les événements pluvieux n'ont pas été pris en compte dans cette série,

\begin{tabular}{|c|c|c|c|c|c|c|c|c|c|}
\hline \multicolumn{5}{|c|}{$\begin{array}{l}\text { PERRODE NORMALE } \\
\text { (d'octobre d avril) }\end{array}$} & \multicolumn{5}{|c|}{$\begin{array}{l}\text { PERIODE CRITIQUE } \\
\text { (de mai à septembre) }\end{array}$} \\
\hline Classe & $\begin{array}{l}\text { effectif de } \\
\text { la classe }\end{array}$ & $\begin{array}{c}\text { corrélation } \\
(d, h)\end{array}$ & $\begin{array}{c}\text { corrélation } \\
(d, T S L)\end{array}$ & $\begin{array}{l}\text { corrélation } \\
(h, T S L)\end{array}$ & Classe & $\begin{array}{l}\text { effectif de } \\
\text { la classe }\end{array}$ & $\begin{array}{c}\text { corrélation } \\
(d, h)\end{array}$ & $\begin{array}{l}\text { corrélation } \\
(d, T S L)\end{array}$ & $\begin{array}{c}\text { corrélation } \\
(h, T S L)\end{array}$ \\
\hline 1 & 13 & $-0,215$ & $-0,374$ & 0,016 & 2 & 11 & 0,084 & $-0,048$ & 0,613 \\
\hline 2 & 27 & 0,789 & 0,140 & 0,182 & 3 & 113 & $-0,075$ & $-0,141$ & $-0,066$ \\
\hline 4 & 111 & 0,504 & 0,091 & $-0,047$ & 4 & 22 & $-0,094$ & $-0,096$ & $-0,433$ \\
\hline 5 & 53 & 0,030 & 0,203 & $-0,121$ & 7 & 55 & $-0,018$ & $-0,227$ & 0,226 \\
\hline 6 & 9 & 0,430 & 0,738 & 0,413 & & & & & \\
\hline
\end{tabular}

Tableau 3 : Valeur des corrélations entre les couples de variable $(d, h),(d, T S L)$, et $(h, T S L)$. 


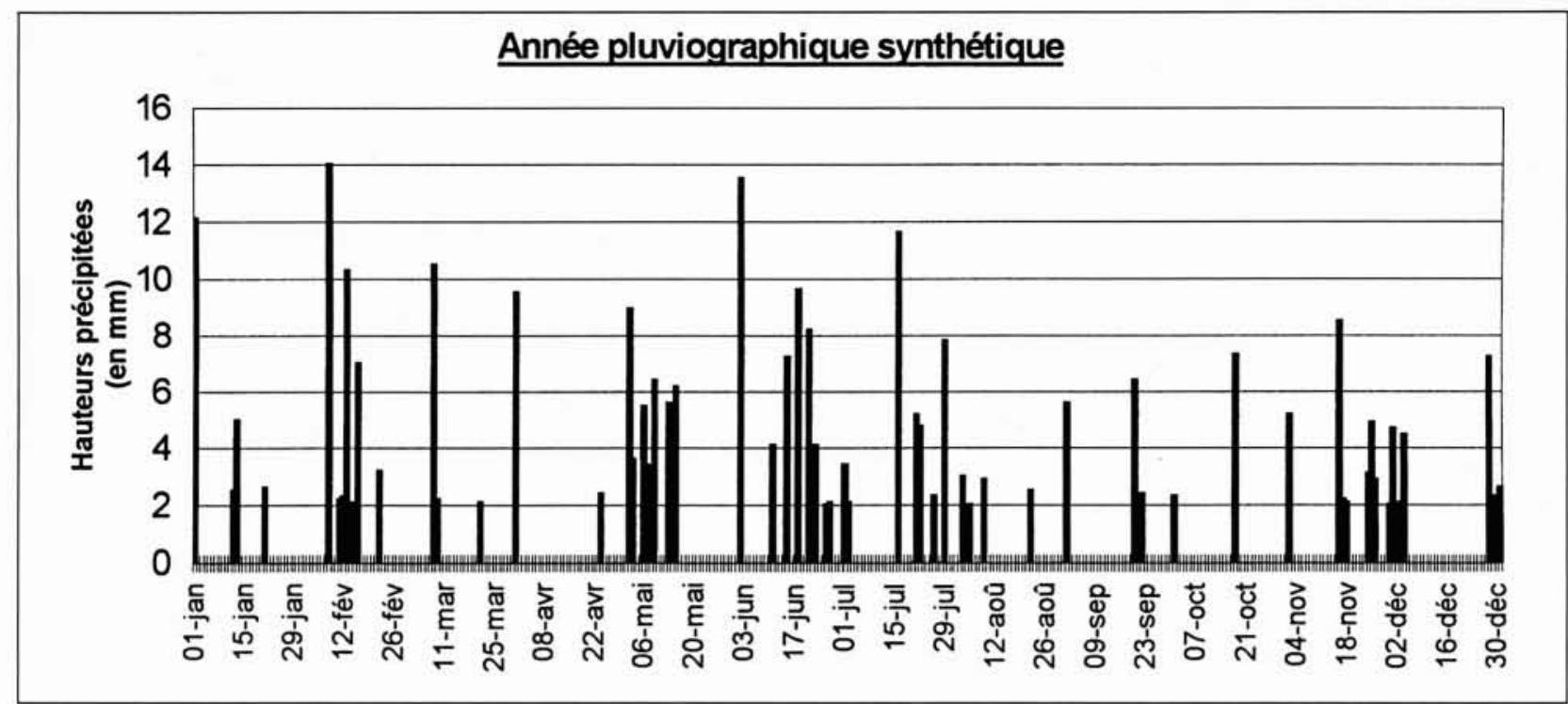

2. Année pluviographique synthétique

en particulier les événements à caractère exceptionnel, situés dans les classes à faible probabilité d'apparition, qui ont été exclus par le procédé de tirage au sort pour la constitution de l'année synthétique. De même, les épisodes pluvieux n'ayant, a priori, pas provoqué de ruissellement (hauteurs précipitées $<2 \mathrm{~mm}$ ) n'ont pas été pris en compte.

Cette année synthétique est donc représentative d'une certaine pluviométrie, caractérisée par des pluies d'intensité moyenne, ayant provoqué ruissellement. La notion de succession dans le temps (à l'échelle d'une année) de ces événements pluvieux, et des périodes de temps sec leur étant associées est ici essentielle. Il faut là encore replacer cette analyse dans le contexte de l'étude générale, qui vise à mettre en évidence les facteurs susceptibles de contribuer aux différents niveaux de pollution. L'année synthétique permettra de simuler les conditions pluviométriques pouvant générer des effets chroniques et/ou cumulatifs sur le milieu récepteur.

On notera que si l'année ainsi produite est une année médiane en termes d'occurrence des différentes classes et en paramètres descriptifs (durée, hauteur), elle comporte tout de même des épisodes pluvieux " extrêmes " (appartenant à des classes à précipitations abondantes par exemple).

Le phénomène de lissage qui apparaît à l'observation du graphique représentant l'année synthétique est donc directement lié à la nature de l'échantillon (figure 2).

\section{VI $\square$ CONCLUSION}

La méthodologie développée dans cette étude a permis de générer une "année synthétique" en exploitant les résultats obtenus lors de la phase de caractérisation des événements (inventaire, classification, répartition du temps sec). La valeur du seuil de précipitations retenu, ainsi que la détermination de la fin d'un événement définie par l'occurrence de temps sec influent directement sur le nombre et la nature des événements à traiter. Par conséquent, l'année synthétique repose sur des critères de définition basés sur des considérations a priori (notions de début et de fin d'événement notamment) liées à la zone d'étude.

Sur le plan météorologique, cette approche s'est révélée tout à fait satisfaisante, dans la mesure où les résultats obtenus sont aisément interprétables pour une population d'événements pluvieux présentant une grande variété de comportements.

L'intérêt de cette méthodologie est qu'elle offre une représentation de la succession dans le temps des événements pluvieux et des périodes de temps sec associées, à l'échelle annuelle. Cela permettra d'appréhender, grâce aux modèles de qualité, les effets chroniques et cumulatifs de la pollution générée par les rejets de temps de pluie. Les effets aigus pourront quant à eux être appréciés à partir des classes de pluie obtenues lors de la première partie de l'étude.

\section{BIBLIOGRAPHIE}

[1] ANJOU-RECHERCHE, 1996. Etude méthodologique de l'impact des déversements en temps de pluie : application à la rivière Orne (57). Rapport de synthèse : Agence de l'Eau Rhin-Meuse, mai 1996.

[2] ANJOU-RECHERCHE, 1995. Etude méthodologique de l'impact des déversements en temps de pluie : application à la rivière Orne (57). Module AS, éléments AS1 et AS2, analyse du site et analyse pluviométrique. Rapport de module : Agence de l'Eau Rhin-Meuse, octobre 1995.

[3] DER MEGREDITCHIAN G. Le traitement statistique des données multidimensionnelles : application à la météorologie. Publication de Météo France, série cours et manuels $\mathrm{N}^{\circ} 8$.

[4] JAMBU et LEBEAUX, 1978. Classification automatique pour l'analyse des données. Dunod, 1978.

[5] LABORATOIRE D'HYDROLOGIE MATHEMATIQUE, 1983. Guide de construction et d'utilisation des pluies de projet. Rapport: L.H.M. Montpellier, octobre 1983.

[6] LEBART, MORINEAU et FENELON, 1979. Traitement des données statistiques. Dunod, 1979.

[7] LEBART, MORINEAU et TABARD, 1979. Techniques de la description statistique. Dunod, 1979.

[8] MISSION DELEGUEE DE BASSIN RHIN-MEUSE, 1981. Hauteurs des précipitations journalières décennales de la France du Nord-Est. Carte et note explicative, juin 1981.

[9] ROUX C., 1996. Analyse des précipitations en hydrologie urbaine. Exemple de la Seine-Saint-Denis. Thèse : Ecole Nationale des Ponts et Chaussées, 1996. 